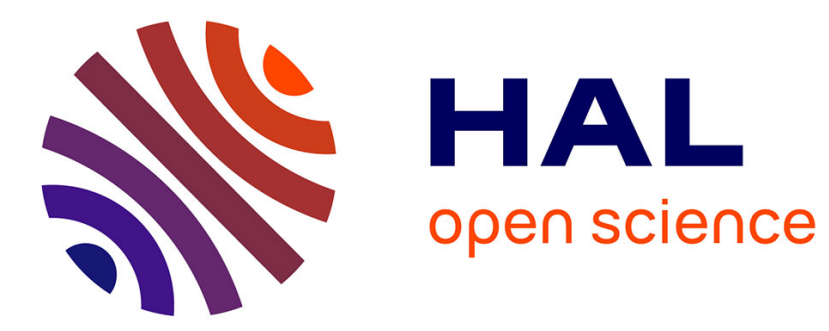

\title{
A study of the dynamic of influence through differential equations
}

\author{
Emmanuel Maruani, Michel Grabisch, Agnieszka Rusinowska
}

\section{To cite this version:}

Emmanuel Maruani, Michel Grabisch, Agnieszka Rusinowska. A study of the dynamic of influence through differential equations. RAIRO - Operations Research, 2012, 46 (1), pp.83-106. 10.1051/ro/2012009 . halshs-00699012

\section{HAL Id: halshs-00699012 https://shs.hal.science/halshs-00699012}

Submitted on 18 May 2012

HAL is a multi-disciplinary open access archive for the deposit and dissemination of scientific research documents, whether they are published or not. The documents may come from teaching and research institutions in France or abroad, or from public or private research centers.
L'archive ouverte pluridisciplinaire HAL, est destinée au dépôt et à la diffusion de documents scientifiques de niveau recherche, publiés ou non, émanant des établissements d'enseignement et de recherche français ou étrangers, des laboratoires publics ou privés. 


\title{
A study of the dynamic of influence through differential equations ${ }^{\star}$
}

\author{
EMMANUEL MARUANI ${ }^{1}$, MICHEL GRABISCH ${ }^{2}$, and AGNIESZKA \\ RUSINOWSKA $^{3}$ \\ 1 Nomura International, London \\ emmanuel.maruani@gmail.com \\ 2 Paris School of Economics, Université Paris I Panthéon-Sorbonne \\ 106-112 Bd de l'Hôpital, 75647 Paris Cedex 13, France \\ Phone: 0033144078 285, Fax: 0033144078301 \\ michel.grabisch@univ-paris1.fr \\ 3 Paris School of Economics - CNRS, Centre d'Economie de la Sorbonne \\ agnieszka.rusinowska@univ-paris1.fr
}

\begin{abstract}
The paper concerns a model of influence in which agents make their decisions on a certain issue. We assume that each agent is inclined to make a particular decision, but due to a possible influence of the others, his final decision may be different from his initial inclination. Since in reality the influence does not necessarily stop after one step, but may iterate, we present a model which allows us to study the dynamic of influence. An innovative and important element of the model with respect to other studies of this influence framework is the introduction of weights reflecting the importance that one agent gives to the others. These importance weights can be positive, negative or equal to zero, which corresponds to the stimulation of the agent by the 'weighted' one, the inhibition, or the absence of relation between the two agents in question, respectively. The exhortation obtained by an agent is defined by the weighted sum of the opinions received by all agents, and the updating rule is based on the sign of the exhortation. The use of continuous variables permits the application of differential equations systems to the analysis of the convergence of agents' decisions in long-time. We study the dynamic of some influence functions introduced originally in the discrete model, e.g., the majority and guru influence functions, but the approach allows the study of new concepts, like e.g. the weighted majority function. In the dynamic framework, we describe necessary and sufficient conditions for an agent to be follower of a coalition, and for a set to be the boss set or the approval set of an agent.
\end{abstract}

JEL Classification: C7, C6, D7

Keywords: social network, inclination, importance weight, decision, influence function, differential equations

Corresponding author: Michel Grabisch

\section{Introduction}

The phenomenon of influence between individuals or, more broadly, influence of a group of agents on an individual, is studied carefully in numerous works, e.g., in psychology, sociology, economics, physics and mathematics. The economics literature offers several surveys of different models of influence and of different approaches to this phenomenon; see, e.g., Jackson (2008), Grabisch and Rusinowska (2010c), Rusinowska (2010).

\footnotetext{
* Emmanuel Maruani was a student at the Université de Paris 1 and Ecole Nationale des Ponts et Chaussées when this research has been conducted. Michel Grabisch and Agnieszka Rusinowska acknowledge support by the National Agency for Research (Agence Nationale de la Recherche), Reference: ANR-09-BLAN-0321-01.
} 
Some of the works on influence and interaction, in particular in the game theoretical literature, are one-step models. In reality, however, the influence does not necessarily stop after one step, but may iterate. Consequently, in order to study the iteration of influence, different dynamic models of influence are proposed by sociologists and economists. One of the leading works of opinion formation is introduced in DeGroot (1974). In that model, individuals start with initial opinions on a subject and place some (nonnegative) weights on the current beliefs of other agents in forming their own beliefs for the next period. These beliefs are updated over time. Several other authors study the DeGroot model and propose its generalizations, in particular, models in which the updating of beliefs can vary in time and circumstances; see e.g. Berger (1981), DeMarzo et al. (2003), Krause (2000), Lorenz (2005), Friedkin and Johnsen (1990, 1997), Jackson (2008), Golub and Jackson (2010). A related dynamic framework of influence is also presented in Asavathiratham (2000), Asavathiratham et al. (2001) and Koster et al. (2010).

A cooperative approach to influence is presented in $\mathrm{Hu}$ and Shapley (2003a,b), where the command structure is applied to model players' interaction relations by simple games. Boss sets and approval sets for a player are defined, and based on these sets a simple game called the command game for the player is constructed. Also the concept of command function is defined. The authors introduce an authority distribution over an organization and define the authority equilibrium equation. In particular, they consider multi-step commands, where commands can be implemented through command channels.

The present paper is related to another framework of influence originally introduced in Hoede and Bakker (1982). In the original one-step model, agents have to make their acceptance-rejection decision on a certain issue. Each agent has an inclination to say either 'yes' or 'no', but due to a possible influence of the other agents, his final decision ('yes' or 'no') may be different from his initial inclination. Refinements and generalizations of this model are presented in several works. Grabisch and Rusinowska (2010a) investigate some tools to analyze influence in this framework. In particular, they define the influence indices to measure the influence of a coalition on an agent, introduce several influence functions and study their properties, and investigate the concept of a follower of a coalition. In Grabisch and Rusinowska (2010b) the yes-no model of influence is generalized to a framework in which each agent has an ordered set of possible actions, and in Grabisch and Rusinowska (2011b) the model is extended to a continuum of actions. Grabisch and Rusinowska (2009) compare the influence model with the framework of simple games called command games ( $\mathrm{Hu}$ and Shapley $(2003 \mathrm{~b}, \mathrm{a})$ ). It is shown that the presented framework of influence is more general than the framework of command games. In Grabisch and Rusinowska (2011a) the exact relations between the key concepts of the influence model and the framework of command games are established. There are also some studies of the dynamic of influence in the model mentioned above, i.e., the model of initial inclinations and final decisions. In Grabisch and Rusinowska (2011c) the yes-no model with a single step of mutual influence is generalized to a dynamic model of influence based on aggregation functions. The decision process in which the mutual influence does not stop after one step but iterates, and the convergence of an influence function are studied. In particular, the authors investigate stochastic influence functions and apply Markov chains theory to the analysis of such functions.

The aim of the present paper is to apply another approach proposed in Maruani (2010) to study the dynamic of influence. We propose a dynamic model in which an agent gives 
a certain importance, reflected by a weight, to other agents in making his final decision. Such a weight might be positive, negative, or equal to zero, which corresponds to the stimulation of the agent by the 'weighted' one, the inhibition, and the absence of relation between the agents in question, respectively. Furthermore, we define the exhortation of an agent which is the weighted sum of the opinions that the agent receives from all agents. The opinions of the agents are updated over time and the updating rule is based on the sign of the exhortation. The use of continuous variables permits the application of differential equations systems to the analysis of the convergence of agents' decisions in long-time. We study the dynamic of some influence functions introduced originally in the discrete model, e.g., the majority and guru influence functions, but the approach allows the study of new concepts, like e.g. the weighted majority function. In the dynamic framework, we describe necessary and sufficient conditions for an agent to be follower of a coalition, and for a set to be the boss set or the approval set of an agent. By applying the approach based on differential equations to the influence model, we recover the results of the discrete model on classical influence functions, and the results on the boss and approval sets for command games equivalent to some selected influence functions.

What are the innovative elements of the model with respect to other studies of the influence framework, and what are the improvements and advantages that the model brings to the analysis of influence? First of all, we like to stress that to the best of our knowledge the approach based on differential equations has not been used in the influence frameworks so far. We see two original features of the present model compared to our previous studies as well as to other ones.

First, the main aim is the analysis of the dynamic aspects of influence, where the opinion of agents is evolving in time, instead of considering as in our studies so far that influence stops after one step. Generally speaking, there are two main streams in analyzing the dynamic aspects of a system. The first one is to model the system with a set of states, and assume a probabilistic transition between states. This leads to stachastic processes, and in particular, Markovian processes. In a recent paper, Grabisch and Rusinowska (2011c) follow this line and provide a general analysis of convergence of the dynamic yesno model of influence, assuming that agents update their opinion by aggregating opinions of the other agents. The second stream is to establish a set of differential equations governing the evolution of the system. This is the way we follow in this paper. We believe that new insights in the analysis of influence can be brought by this approach. This paper constitutes a first step in this direction.

Its second original feature lies in incorporating the importance weights of positive and negative signs. This allows to model the positive (direct) and negative (opposite) influence at the same time, and in a very simple way. The yes-no model introduced in Grabisch and Rusinowska (2010a) and its generalization to the multi-action model presented in Grabisch and Rusinowska (2010b) are one-step models for which the authors define the indices of positive and negative influence separately, and they do not assume any weights of importance. The same remark holds for our study of command games in Grabisch and Rusinowska (2009) and Grabisch and Rusinowska (2011a). Grabisch and Rusinowska (2011c) do provide a general analysis of convergence of the dynamic yes-no model of influence in which every agent updates his opinion according to his aggregation function. However, the nondecreasingness assumption in the definition of an aggregation function implies positive influence, and therefore the model based on 
aggregation functions does not cover a framework of negative influence. The model of DeGroot (1974) and its modifications (for an overview, see e.g., Jackson (2008)) assume that the opinion of an agent is a number in $[0,1]$ and the aggregation is done through a weighted arithmetic mean (convex combination). Players place weights on the current beliefs of the others in forming their own beliefs for the next period, but these weights are nonnegative and no inhibition possibility is incorporated in the model. The convergence of the process (of the updating/interaction matrix) is studied.

The paper is structured as follows. In Section 2 the main concepts of the influence model in question and the framework of command games are presented. In Section 3 we introduce the dynamic model of influence. The dynamic of selected influence functions is studied in Section 4. Section 5 concerns followers in the dynamic model and under selected influence functions. The analysis of the dynamic model in terms of command games, in particular, the determination of the boss and approval sets in the presented framework is delivered in Section 6. Concluding remarks are given in Section 7.

\section{The model of influence and command games}

\subsection{Main concepts of the influence model}

Let us start by mentioning some notations used in the paper. $(1,1, \ldots, 1) \in\{-1,+1\}^{n}$ is denoted by $1_{N},(-1,-1, \ldots,-1) \in\{-1,+1\}^{n}$ by $-1_{N}$, and mixed cases by $\left(-1_{N \backslash S}, 1_{S}\right)$. For every real number $x \in \mathbb{R},\lfloor x\rfloor$ denotes the largest integer not greater than $x$. By $\delta_{i j}$ we denote the Kronecker delta, i.e., $\delta_{i j}=1$ if $i=j$, and 0 otherwise.

In this section we recapitulate main concepts of the one-step yes-no model of influence investigated in Grabisch and Rusinowska (2010a). We consider a social network with the set of all players (agents, voters) denoted by $N:=\{1, \ldots, n\}$. Each player has to make an acceptance-rejection decision concerning a certain issue, and he has an inclination to say either 'yes' (denoted by +1 ) or 'no' (denoted by -1 ). An inclination vector is denoted by $I=\left(I_{1}, \ldots, I_{n}\right)$, where $I_{k} \in\{-1,+1\}$ indicates the inclination of agent $k$, for each $k \in N$. For any $I \in\{-1,+1\}^{n}, I^{+}$denotes the set of players with the positive inclination

$$
I^{+}:=\left\{k \in N: I_{k}=+1\right\} .
$$

Moreover, for any $S \subseteq N$, we denote by $U I_{S}$ the set of all inclination vectors under which all members of $S$ have the same inclination

$$
U I_{S}:=\left\{I \in\{-1,+1\}^{n}: \forall k, j \in S\left[I_{k}=I_{j}\right]\right\} .
$$

In particular, $U I_{k}=\{-1,+1\}^{n}$ for any $k \in N$. We denote by $I_{S}$ the value $I_{k}$ for some $k \in S, I \in U I_{S}$.

It is assumed that agents may influence each other in the network, and due to the influences the final decision of an agent may be different from his original inclination. Formally, each inclination vector $I \in\{-1,+1\}^{n}$ is transformed into a decision vector $B(I)=\left(B_{1}(I), \ldots, B_{n}(I)\right)$, where $B:\{-1,+1\}^{n} \rightarrow\{-1,+1\}^{n}, I \mapsto B(I)$ is the influence function $^{1}$, and $B_{k}(I)$ indicates the decision made by agent $k$, for each $k \in N$. The set of all influence functions is denoted by $\mathcal{B}$.

\footnotetext{
${ }^{1}$ We can also speak of the influence function of agent $k, B_{k}:\{-1,+1\}^{n} \rightarrow\{-1,+1\}, I \mapsto B_{k}(I)$, for each $k \in N$.
} 
One of the key concepts of the influence model is the concept of follower. An agent is said to be follower of a coalition if he always decides according to the inclination of that coalition, assuming that the coalition in question is unanimously inclined. Formally, for $\emptyset \neq S \subseteq N$ and $B \in \mathcal{B}$, the set of followers of $S$ under $B$ is therefore defined as

$$
F_{B}(S):=\left\{j \in N: \forall I \in U I_{S}\left[B_{j}(I)=I_{S}\right]\right\}
$$

We recapitulate three particular influence functions that have been introduced and investigated in Grabisch and Rusinowska (2010a):

- Let $n \geq t>\left\lfloor\frac{n}{2}\right\rfloor$. The majority influence function $\mathrm{Maj}^{[t]} \in \mathcal{B}$ is defined by

$$
\operatorname{Maj}^{[t]}(I):=\left\{\begin{array}{ll}
1_{N}, & \text { if }\left|I^{+}\right| \geq t \\
-1_{N}, & \text { if }\left|I^{+}\right|<t
\end{array}, \quad \forall I \in\{-1,+1\}^{n} .\right.
$$

According to the majority influence function if a majority of players has the positive inclination, then all agents decide +1 , otherwise all decide -1 . For each $S \subseteq N$, the set of followers under the majority function is equal to

$$
F_{\mathrm{Maj}[t]}(S)= \begin{cases}N, & \text { if }|S| \geq t \\ \emptyset, & \text { if }|S|<t\end{cases}
$$

i.e., everybody follows a coalition with a cardinality of at least $t$, and nobody follows a coalition with less than $t$ members.

- Let $\widetilde{k} \in N$ be a particular player called the guru. The guru influence function Gur $^{[\widetilde{k}]} \in$ $\mathcal{B}$ is defined by

$$
\operatorname{Gur}_{j}^{[\widetilde{k}]}(I):=I_{\widetilde{k}}, \quad \forall I \in\{-1,+1\}^{n}, \quad \forall j \in N .
$$

Hence, according to this function, when a guru exists, every agent follows the guru. For each $S \subseteq N$, the set of followers under the guru function is given by

$$
F_{\text {Gur }[\tilde{k}]}(S)= \begin{cases}N, & \text { if } \widetilde{k} \in S \\ \emptyset, & \text { if } \widetilde{k} \notin S .\end{cases}
$$

In other words, all agents follow a coalition containing the guru, and nobody follows a coalition without the guru.

- The identity function $\mathrm{Id} \in \mathcal{B}$ depicts the absence of any influence and is defined by

$$
\operatorname{Id}(I):=I, \quad \forall I \in\{-1,+1\}^{n} .
$$

Moreover, we have for each $S \subseteq N$,

$$
F_{\mathrm{ld}}(S)=S,
$$

which means that all members of a coalition and only them follow that coalition. 


\subsection{Command games and equivalence with influence functions}

Next, we present some of the main concepts concerning command games that have originally been introduced by $\mathrm{Hu}$ and Shapley $(2003 \mathrm{a}, \mathrm{b})$.

Let $N=\{1, \ldots, n\}$ be the set of agents (players, voters). With each $k \in N$, two collections of coalitions, the so called boss set and approval set are associated. Hu and Shapley (2003a) define these sets as follows. For $k \in N$ and $S \subseteq N \backslash k$ :

- $S$ is a boss set for $k$ if $S$ determines the choice of $k$;

- $S$ is an approval set for $k$ if $k$ can act with the approval of $S$.

It is assumed that any superset (in $N \backslash k$ ) of a boss set is a boss set. For each $k \in N$, a simple game $\left(N, \mathcal{W}_{k}\right)$ called the command game for $k$ is created, with the set of winning coalitions given by

$$
\mathcal{W}_{k}:=\{S: S \text { is a boss set for } k\} \cup\{S \cup k: S \text { is a boss or approval set for } k\} \text {. }
$$

We recover the boss sets for agent $k$

$$
\text { Boss }_{k}=\left\{S \subseteq N \backslash k: S \in \mathcal{W}_{k}\right\}=\mathcal{W}_{k} \cap 2^{N \backslash k}
$$

and the approval sets for $k$

$$
A p p_{k}=\left\{S \subseteq N \backslash k: S \cup k \in \mathcal{W}_{k} \text { but } S \notin \mathcal{W}_{k}\right\}
$$

Obviously, Boss $_{k} \cap A p p_{k}=\emptyset$. Given the set of command games $\Omega=\left\{\left(N, \mathcal{W}_{k}\right): k \in N\right\}$, for any coalition $S \subseteq N$, the command function $\omega(S)$ is defined as the set of all members that are commandable by $S$ :

$$
\omega(S):=\left\{k \in N: S \in \mathcal{W}_{k}\right\}
$$

In Grabisch and Rusinowska (2009) the model of influence is applied to the framework of command games and the relations between these two frameworks are shown; see also Grabisch and Rusinowska (2011a). We present one of these relations, i.e., the equivalence between command games and (command) influence functions.

Let $\Omega=\left\{\left(N, \mathcal{W}_{k}\right): k \in N\right\}$ be a set of command games, $\omega(S)$ be a set of agents commandable by $S$, and $F_{B}(S)$ denote the set of followers of $S$ under an influence function $B$. The influence function $B$ and the set of command games $\Omega$ are said to be equivalent if $F_{B} \equiv \omega$, i.e., if for each coalition $S \subseteq N$, the set of followers of $S$ under the influence function $B$ and the set of agents commandable by $S$ under $\Omega$ coincide.

In Grabisch and Rusinowska (2009) we construct command games equivalent to the influence functions recapitulated in Section 2.1 and determine boss and approval sets for these command games:

(i) Let $n \geq t>\left\lfloor\frac{n}{2}\right\rfloor$ and $\mathrm{Maj}^{[t]} \in \mathcal{B}$ be the majority influence function and let $\left\{\left(N, \mathcal{W}_{k}^{\mathrm{Maj}}{ }^{[t]}\right)\right.$ : $k \in N\}$ be a set of command games given by

$$
\mathcal{W}_{k}^{\mathrm{Maj}^{[t]}}=\{S \subseteq N:|S| \geq t\}, \forall k \in N
$$


The majority influence function $\mathrm{Maj}^{[t]}$ and the set of command games $\left\{\left(N, \mathcal{W}_{k}^{\mathrm{Maj}^{[t]}}\right)\right.$ : $k \in N\}$ are equivalent. In other words, the command games in which winning coalitions for each player are the ones with the cardinality at least $t, n \geq t>\left\lfloor\frac{n}{2}\right\rfloor$, are equivalent to Maj ${ }^{[t]}$. Moreover, we have for $n>2, n \geq t>\left\lfloor\frac{n}{2}\right\rfloor$, and $k \in N$

$$
\begin{gathered}
\operatorname{Boss}_{k}^{\mathrm{Maj}^{[t]}}=\{S \subseteq N:|S| \geq t \wedge k \notin S\} \\
A p p_{k}^{\mathrm{Maj}^{[t]}}=\{S \subseteq N:|S|=t-1 \wedge k \notin S\} .
\end{gathered}
$$

In particular, for $t=n, k \in N$,

$$
\operatorname{Boss}_{k}^{\mathrm{Maj}^{[t]}}=\emptyset, \quad A p p_{k}^{\mathrm{Maj}^{[t]}}=N \backslash k .
$$

(ii) Let Gur ${ }^{[\widetilde{k}]} \in \mathcal{B}$ be the guru function with the guru $\widetilde{k} \in N$ and let $\left\{\left(N, \mathcal{W}_{k}^{\text {Gur }}{ }^{[\widetilde{k}]}\right): k \in N\right\}$ be a set of command games given by

$$
\mathcal{W}_{k}^{\text {Gur }}{ }^{[\tilde{k}]}=\{S \subseteq N: \widetilde{k} \in S\}, \forall k \in N
$$

The guru function Gur ${ }^{[\widetilde{k}]}$ and the set of command games $\left\{\left(N, \mathcal{W}_{k}^{\text {Gur }}{ }^{[\tilde{k}]}\right): k \in N\right\}$ are equivalent. Hence, the command games in which winning coalitions for each player are the coalitions containing a certain player $\widetilde{k}$, are equivalent to the guru function Gur ${ }^{[\widetilde{k}]}$ with the guru $\widetilde{k}$. Moreover,

$$
\begin{gathered}
\operatorname{Boss}_{\widetilde{k}}^{\text {Gur }^{[\tilde{k}]}}=\emptyset, \quad \operatorname{App}_{\widetilde{k}}^{\text {Gur }^{[\widetilde{k}]}}=2^{N \backslash \widetilde{k}} \\
\operatorname{Boss}_{k}^{\text {Gur }}{ }^{[\widetilde{k}]}=\{S \subseteq N: \widetilde{k} \in S \wedge k \notin S\}, \quad A p p_{k}^{\text {Gur }^{[\widetilde{k}]}}=\emptyset, \quad \text { for } k \neq \widetilde{k} .
\end{gathered}
$$

(iii) Let $\mathrm{Id} \in \mathcal{B}$ be the identity function and let $\left\{\left(N, \mathcal{W}_{k}^{\text {ld }}\right): k \in N\right\}$ be a set of command games given by

$$
\mathcal{W}_{k}^{\text {ld }}=\{S \subseteq N: k \in S\}, \forall k \in N
$$

The identity function Id and the set of command games $\left\{\left(N, \mathcal{W}_{k}^{\text {ld }}\right): k \in N\right\}$ are equivalent. This means that the command games, in which for each player $k$ winning coalitions for $k$ are the coalitions containing $k$, are equivalent to the identity function. We have also for each $k \in N$

$$
\operatorname{Boss}_{k}^{\text {ld }}=\emptyset, \quad A p p_{k}^{\text {ld }}=2^{N \backslash k} .
$$

\section{The dynamic model of influence}

\subsection{Description of the model and stable states}

In order to analyze the dynamic aspects of influence, we consider the following model introduced originally in Maruani (2010).

Let $N=\{1,2, \ldots, n\}$ denote the set of agents. We are interested in the influence of all $n$ agents on a player $i \in N$. Let $e_{j}$ denote the inclination of agent $j \in N$, where $e_{j}= \pm 1$. Agent $i$ gives to agent $j$ a certain importance which is reflected by a weight $p_{i j} \in[-1,1]$. $p_{i j}>0$ corresponds to the stimulation of $i$ by $j, p_{i j}<0$ corresponds to the inhibition, 
and $p_{i j}=0$ means the absence of relation. The state (inclination) $e_{j}$ will contribute to the decision of agent $i$ with the weight $c_{i j}=p_{i j} e_{j}$.

What is the interpretation of the contribution weights $c_{i j}$ ? Obviously, they can be positive, negative or zero, which means that if player $i$ would consult only the state $e_{j}$, then $i$ 's decision would be 'yes', 'no' or would be equal to $i$ 's state, respectively. More precisely, suppose that agent $i$ when forming his decision does not take into account the inclination of agent $j$, i.e., $p_{i j}=0$. Then obviously $p_{i j} e_{j}=0$, independently of the inclination of agent $j$, which reflects no contribution of $j$ 's state to $i$ 's decision. Suppose that $j$ stimulates the decision of agent $i$, i.e., $p_{i j}>0$. Then the weight of the contribution of $j$ 's state to the decision of agent $i$ has the same sign as $e_{j}$, which means that $i$ 's decision would be as the state of $j$ when $i$ considered only the state $e_{j}$. Moreover, the higher $p_{i j}$ the more $e_{j}$ contributes to $i$ 's decision being the same as $e_{j}$ when all players are considered. Suppose now that $j$ inhibits or discourages the decision of agent $i$, i.e., $p_{i j}<0$. Then the weight of the contribution of $j$ 's state to the decision of agent $i$ has the opposite sign than $e_{j}$, which means that $i$ 's decision would be opposite to the state of $j$ when $i$ considered only the state $e_{j}$. In particular, if $j$ is inclined negatively, then $i$ 's decision would be positive when consulting only $e_{j}$. The higher the absolute value of $p_{i j}$ the more $e_{j}$ contributes to $i$ 's decision being opposite to $e_{j}$ when all players are considered.

Since the players interact, every agent consults the states of all agents, and based on the importance weights he aggregates all contributions to his decision. Hence, the exhortation $E_{i}$ obtained by agent $i \in N$ is defined by the weighted sum of the inclinations of all agents, or saying differently, by the weighted sum of the opinions that $i$ receives from the agents (including his own opinion):

$$
E_{i}=\sum_{j \in N} c_{i j}=\sum_{j \in N} p_{i j} e_{j} .
$$

Figure 1 presents the idea of the exhortation.

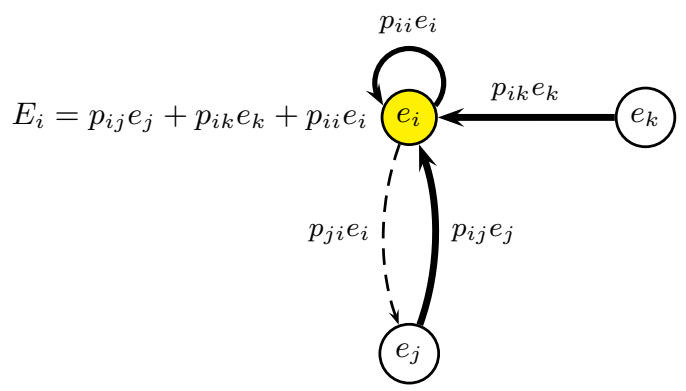

Fig. 1. The exhortation

The updating rule is the following. If $E_{i}>0$, then agent $i$ goes to the state +1 . If $E_{i}<0$, then he goes to the state -1 , and if $E_{i}=0$, then $i$ stays in his present state.

The influence function $B_{i}$ of agent $i \in N$ is defined by

$$
B_{i}(e)=\operatorname{sgn}\left(E_{i}\right), \text { where } e=\left(e_{1}, \ldots, e_{n}\right) \text { and } \operatorname{sign}\left(E_{i}\right)= \begin{cases}1, & \text { if } E_{i}>0 \\ -1, & \text { if } E_{i}<0 \\ e_{i}, & \text { if } E_{i}=0\end{cases}
$$


Remark 1 In this dynamic model, we can express the influence functions recapitulated in Section 2.1 as follows:

(i) The majority influence function with $t=\left\lfloor\frac{n}{2}\right\rfloor+1$ in which every agent plays a role can be represented by $p_{i j}=\frac{1}{n}$.

(ii) The guru function with the guru $\widetilde{k}$ in which every agent is influenced only by the guru can be represented by $p_{i j}=0$ for each $j \neq \widetilde{k}$ and $p_{i \widetilde{k}}=1$.

(iii) The identity function in which every agent influences only himself can be represented by $p_{i j}=\delta_{i j}$.

Stable states of the system satisfy $e_{i}^{(k+1)}=e_{i}^{(k)}$ for each $i \in N$, starting from a certain $k$, where $k$ is the number of iteration and $e_{i}^{(k)}$ denotes the inclination (state) of $i$ at time $k$. This means that the state $e_{i}$ and the exhortation $E_{i}$ have the same sign, and therefore $e_{i} E_{i}>0$. In other words, the stable states satisfy the following inequality:

$$
\sum_{j \in N} p_{i j} e_{i} e_{j}>0 \text { for each } i \in N \text {. }
$$

The evolution of the population can be expressed by

$$
e_{i}^{(k+1)}=B_{i}\left(e_{1}^{(k)}, e_{2}^{(k)}, \ldots, e_{j}^{(k)}, \ldots, e_{n}^{(k)}\right)
$$

where $B_{i}$ is the influence function of voter $i \in N$. A stable state satisfies therefore

$$
e_{i}^{(k)}=B_{i}\left(e_{1}^{(k)}, e_{2}^{(k)}, \ldots, e_{j}^{(k)}, \ldots, e_{n}^{(k)}\right) .
$$

An obvious way to get stability is to forbid any relation between agents, i.e., to assign the weights as $p_{i j}=\delta_{i j}$.

\subsection{Dynamic of the model in the continuous framework}

Let us consider an extension of the dynamic influence model to the continuous framework; see also Maruani (2010). The influence function remains

$$
e_{i}^{(k+1)}=\operatorname{sgn}\left(E_{i}^{(k)}\right)
$$

which together with $E_{i}^{(k)}=\sum_{j \in N} p_{i j} e_{j}^{(k)}$ can be written as

$$
\frac{e_{i}^{(k+1)}-e_{i}^{(k)}}{(k+1)-k}=\operatorname{sgn}\left(\sum_{j} p_{i j} e_{j}^{(k)}\right)-e_{i}^{(k)} .
$$

For a small time step, we can make the approximation

$$
\frac{e_{i}^{(k+1)}-e_{i}^{(k)}}{(k+1)-k} \approx \frac{d e_{i}}{d t}
$$

and also the approximation

$$
\operatorname{sgn}\left(\sum_{j} p_{i j} e_{j}\right) \approx \tanh \left(a \sum_{j} p_{i j} e_{j}\right)
$$


where the parameter $a$ controls the tendency towards the function sgn. This approximation keeps the properties of the function sgn that are essential for our analysis: it is increasing and bounded between -1 and 1 and nullifies in 0 .

From (12), (13), and (14), in order to study the dynamic of the model, we need to solve the following system:

$$
\frac{d e_{i}}{d t}+e_{i}=\tanh \left(a \sum_{j} p_{i j} e_{j}\right), \quad i=1, \ldots, n
$$

and then put $e_{i}=\operatorname{sgn}\left[e_{i}(t)\right]$. In Section 4 we apply this dynamic approach of differential equations to classical influence functions. The results that we present have been originally shown in Maruani (2010).

\section{The dynamic of selected influence functions}

\subsection{The majority influence function with $t=\left\lfloor\frac{n}{2}\right\rfloor+1$}

As mentioned in Remark 1(i), the majority influence function with $t=\left\lfloor\frac{n}{2}\right\rfloor+1$ can be represented by the weights $p_{i j}=\frac{1}{n}$. The system (15) of differential equations that we need to solve is therefore

$$
\frac{d e_{i}}{d t}+e_{i}=\tanh \left(\frac{a}{n} \sum_{j} e_{j}\right), \quad i=1, \ldots, n .
$$

The dynamic approach allows for the analysis of the influence model introduced in Grabisch and Rusinowska (2011b) in which agents have a continuum of actions, i.e., the set of all inclinations is equal to an interval $[x, y]$, where $x, y \in \mathbb{R}$.

Lemma 1 For $a>1$, the differential equation $\frac{d x}{d t}+x=\tanh ($ ax $)$ has an unstable fixed point in 0 and another stable fixed point.

Proof: In the equation

$$
\frac{d x}{d t}+x=\tanh (a x)
$$

we put $a x=y$. Hence,

$$
\frac{d y}{d t}+y=a \tanh (y)=\frac{1}{p} \tanh (y)
$$

with $p=\frac{1}{a}$. The fixed points satisfy $\tanh (y)=p y$. There is always $y=0$ and another fixed point $y_{0}$ iff $p<1(a>1)$. We are interested in the stability of fixed points. In the neighborhood of $y=0$

$$
\begin{gathered}
\frac{d y}{d t}+y=\frac{1}{p} \tanh (y) \approx \frac{y}{p} \\
\frac{d y}{d t}+\frac{p-1}{p} y=0 \\
y(t)=y(0) \exp \frac{1-p}{p} t
\end{gathered}
$$


For $0<p<1$ the origin is unstable.

In the neighborhood of $y_{0}$, we put $y(t)=y_{0}+\varepsilon(t)$

$$
\begin{aligned}
\frac{d y}{d t} & +y=\frac{1}{p} \tanh (y) \\
\frac{d \varepsilon}{d t}+y_{0}+\varepsilon(t) & =\frac{1}{p} \tanh \left(y_{0}+\varepsilon(t)\right) \\
& =\frac{1}{p} \frac{\tanh \left(y_{0}\right)+\tanh (\varepsilon(t))}{1+\tanh \left(y_{0}\right) \tanh (\varepsilon(t))} \\
& =\frac{1}{p} \frac{p y_{0}+\tanh (\varepsilon(t))}{1+p y_{0} \tanh (\varepsilon(t))}
\end{aligned}
$$

where we have used $p y_{0}=\tanh \left(y_{0}\right)$. A first order approximation yields

$$
\begin{aligned}
\frac{d \varepsilon}{d t}+y_{0}+\varepsilon(t) & \approx \frac{1}{p} \frac{p y_{0}+\varepsilon(t)}{1+p y_{0} \varepsilon(t)} \approx \frac{1}{p}\left[p y_{0}+\varepsilon(t)\right]\left[1-p y_{0} \varepsilon(t)\right] \\
& \approx y_{0}-p y_{0}^{2} \varepsilon(t)+\frac{\varepsilon(t)}{p}
\end{aligned}
$$

and ultimately

$$
\frac{d \varepsilon}{d t}+\left(1+p y_{0}^{2}-\frac{1}{p}\right) \varepsilon(t)=0
$$

If $\left(1+p y_{0}^{2}-\frac{1}{p}\right)>0$, then the solution is the decreasing exponential and point $y_{0}$ is stable. Let us show that $\left(1+p y_{0}^{2}-\frac{1}{p}\right)>0$. Let

$$
\begin{gathered}
A=1+p y_{0}^{2}-\frac{1}{p} \\
p y_{0}=\tanh \left(y_{0}\right) \\
A=1+y_{0} \tanh \left(y_{0}\right)-\frac{y_{0}}{\tanh \left(y_{0}\right)}=1+y_{0}\left[\tanh \left(y_{0}\right)-\frac{1}{\tanh \left(y_{0}\right)}\right] \\
=1+y_{0}\left[\frac{\tanh ^{2}\left(y_{0}\right)-1}{\tanh \left(y_{0}\right)}\right]=1-y_{0}\left[\frac{1}{\cosh ^{2}\left(y_{0}\right) \tanh \left(y_{0}\right)}\right] \\
=1-y_{0}\left[\frac{1}{\cosh \left(y_{0}\right) \sinh \left(y_{0}\right)}\right]=1-\frac{2 y_{0}}{\sinh \left(2 y_{0}\right)} \\
=1-\frac{u}{\sinh (u)}
\end{gathered}
$$

We have

$$
\begin{gathered}
\frac{\sinh (u)}{u}=1+\frac{u^{2}}{3 !}+\frac{u^{4}}{5 !}+\ldots \\
\frac{\sinh (u)}{u}>1 \Rightarrow 1-\frac{u}{\sinh (u)}>0 .
\end{gathered}
$$


Proposition 1 If agents make their decisions according to the majority influence function with $t=\left\lfloor\frac{n}{2}\right\rfloor+1$, then the decision of each agent converges to the sign of the sum of the agents' inclinations.

Proof: Let us consider the system

$$
\frac{d e_{i}}{d t}+e_{i}=\tanh \left[\frac{a}{n} \sum_{j} e_{j}(t)\right], \quad i=1, \ldots, n .
$$

With $\varepsilon_{i}=\frac{a}{n} e_{i}$, we have, for each $i$,

$$
\frac{d \varepsilon_{i}}{d t}+\varepsilon_{i}=\frac{a}{n} \tanh \left[\sum_{j} \varepsilon_{j}(t)\right]
$$

Adding term by term the equations of the system, the sum $S(t)=\sum_{j} \varepsilon_{j}(t)$ satisfies

$$
\frac{d S}{d t}+S=a \tanh [S(t)]
$$

According to Lemma 1, $S$ converges to a stable fixed point $S_{\infty}$, which satisfies for $a>1$

$$
S_{\infty}=a \tanh \left(S_{\infty}\right)
$$

The system that we consider becomes

$$
\frac{d \varepsilon_{i}}{d t}+\varepsilon_{i}=\frac{a}{n} \tanh [S(t)]
$$

By changing the function $\varepsilon_{i}(t)=E_{i}(t) \exp (-t)\left(\varepsilon_{i}(0)=E_{i}(0)\right)$, we get the equation

$$
\frac{d E_{i}}{d t}=\frac{a}{n} \exp (t) \tanh [S(t)]
$$

whose general solution is

$$
E_{i}(t)=E_{i}(0)+\frac{a}{n} \int_{0}^{t} \exp (u) \tanh [S(u)] d u
$$

The solution for $\varepsilon_{i}$ is therefore

$$
\varepsilon_{i}(t)=\varepsilon_{i}(0) \exp (-t)+\frac{a}{n} \exp (-t) \int_{0}^{t} \exp (u) \tanh [S(u)] d u
$$

We are interested in the asymptotic form of the solution. The first term of the right hand side of (17) vanishes. Let us denote by $\eta_{i}$ the second term, i.e.,

$$
\eta_{i}(t)=\frac{a}{n} \exp (-t) \int_{0}^{t} \exp (u) \tanh [S(u)] d u
$$


Let us fix for the moment some $T$. Then, for $t>T$ we decompose the integral in two terms

$$
\begin{aligned}
\eta_{i}(t) & =\frac{a}{n} \exp (-t)\left[\int_{0}^{T} \exp (u) \tanh [S(u)] d u+\int_{T}^{t} \exp (u) \tanh [S(u)] d u\right] \\
& =\eta_{i}^{(1)}(t)+\eta_{i}^{(2)}(t)
\end{aligned}
$$

and consider successively the two components. We analyze the first term

$$
\eta_{i}^{(1)}(t)=\frac{a}{n} \exp (-t) \int_{0}^{T} \exp (u) \tanh [S(u)] d u
$$

The tanh is bounded by 1 , so we have

$$
\left|\int_{0}^{T} \exp (u) \tanh [S(u)] d u\right| \leq \int_{0}^{T} \exp (u) d u=\exp (T)-1
$$

Given $T, \exp (T)-1$ is a fixed number and we denote it by $B$. Then

$$
\left|\eta_{i}^{(1)}(t)\right| \leq \frac{a B}{n} \exp (-t)
$$

and therefore

$$
\lim _{t \rightarrow \infty} \eta_{i}^{(1)}(t)=0
$$

The second term is

$$
\eta_{i}^{(2)}(t)=\frac{a}{n} \exp (-t) \int_{T}^{t} \exp (u) \tanh [S(u)] d u
$$

For $u$ large enough, which is the case, $S(u)$ is close to $S_{\infty}$. By continuity tanh $[S(u)]$ is close to $\tanh \left(S_{\infty}\right)$, which is equal to $\frac{S_{\infty}}{a}$. It is then appropriate to choose $T$ as follows. Setting

$$
\tanh [S(u)]=\frac{S_{\infty}}{a}[1+\delta(u)]
$$

introduces the function $\delta(u)$ which vanishes at infinity. The free parameter $T$ is then chosen such that for any given positive $\varepsilon$ and for $t>T,|\delta(t)|<\frac{n \varepsilon}{\left|S_{\infty}\right|}$. We have then

$$
\begin{aligned}
\eta_{i}^{(2)}(t) & =\frac{a}{n} \exp (-t) \int_{T}^{t} \exp (u) \tanh [S(u)] d u \\
& =\frac{a}{n} \exp (-t) \int_{T}^{t} \exp (u) \frac{S_{\infty}}{a}[1+\delta(u)] d u \\
& =\frac{S_{\infty}}{n} \exp (-t) \int_{T}^{t} \exp (u) d u+\frac{S_{\infty}}{n} \exp (-t) \int_{T}^{t} \delta(u) \exp (u) d u \\
& =\frac{S_{\infty}}{n}(1-\exp (T-t))+\varphi(t)
\end{aligned}
$$

where the asymptotic value of the first term is $\frac{S_{\infty}}{n}$, and the second term is

$$
\varphi(t)=\frac{S_{\infty}}{n} \exp (-t) \int_{T}^{t} \delta(u) \exp (u) d u
$$




$$
\begin{aligned}
|\varphi(t)| & \leq \frac{S_{\infty}}{n} \exp (-t) \int_{T}^{t} \exp (u) \frac{n \varepsilon}{\left|S_{\infty}\right|} d u \\
& \leq \varepsilon \exp (-t) \int_{T}^{t} \exp (u) d u \\
& \leq \varepsilon
\end{aligned}
$$

The asymptotic value of $\varepsilon_{i}$ is then $\frac{S_{\infty}}{n}$, and the asymptotic value of $\sum_{j} \varepsilon_{j}$ is $\sum_{j=1}^{n} \frac{S_{\infty}}{n}=$ $S_{\infty}$

\section{Remark 2 (The majority vote decreases costs)}

The fixed points of the differential system $\frac{d e_{k}}{d t}=g_{k}\left(e_{1}, e_{2}, \ldots, e_{n}\right), k=1, \ldots, n$ satisfy by definition $\frac{d e_{k}}{d t}=0, k=1, \ldots, n$, which is equivalent to $\sum_{k}\left(\frac{d e_{k}}{d t}\right)^{2}=0$.

Let us suppose the existence of a function $G$ of $n$ variables such that for each $k$

$$
g_{k}\left(e_{1}, e_{2}, \ldots, e_{n}\right)=-\frac{\partial G}{\partial e_{k}}
$$

and calculate

$$
\frac{d G}{d t}=\sum_{k} \frac{\partial G}{\partial e_{k}} \frac{d e_{k}}{d t}=-\sum_{k} g_{k} \frac{d e_{k}}{d t}=-\sum_{k}\left(\frac{d e_{k}}{d t}\right)^{2} \leq 0
$$

which means that $G$ is decreasing in time and reaches its minimum when each $\frac{d e_{k}}{d t}=0$, i.e., for the fixed point of the system. We have

$$
\frac{d e_{k}}{d t}+e_{k}=f_{k}\left(e_{1}, e_{2}, \ldots, e_{n}\right) \Rightarrow \frac{d e_{k}}{d t}=f_{k}\left(e_{1}, e_{2}, \ldots, e_{n}\right)-e_{k} \quad\left(g_{k}=f_{k}-e_{k}\right)
$$

Let us apply this to our differential system (16), letting $\varepsilon_{k}=\frac{a}{n} e_{k}$ :

$$
\frac{d \varepsilon_{k}}{d t}=\frac{a}{n} \tanh \left[\sum_{j} \varepsilon_{j}(t)\right]-\varepsilon_{k}
$$

(all $f$ being identical). We multiply both sides of each differential equation by $\frac{d \varepsilon_{k}}{d t}$

$$
\left(\frac{d \varepsilon_{k}}{d t}\right)^{2}=\frac{a}{n} \frac{d \varepsilon_{k}}{d t} \tanh \left[\sum_{j} \varepsilon_{j}(t)\right]-\varepsilon_{k} \frac{d \varepsilon_{k}}{d t}
$$

and obtain

$$
\sum_{k}\left(\frac{d \varepsilon_{k}}{d t}\right)^{2}=\left\{\frac{a}{n} \tanh \left[\sum_{j} \varepsilon_{j}(t)\right]\right\} \sum_{k} \frac{d \varepsilon_{k}}{d t}-\sum_{k} \varepsilon_{k} \frac{d \varepsilon_{k}}{d t}
$$

With $S(t)=\sum_{k} \varepsilon_{k}(t)$ we have then

$$
\sum_{k}\left(\frac{d \varepsilon_{k}}{d t}\right)^{2}=\frac{a}{n} \frac{d S}{d t} \tanh [S(t)]-\frac{1}{2} \frac{d \sum_{k}\left(\varepsilon_{k}\right)^{2}}{d t}
$$


Note that

$$
\frac{d S}{d t} \tanh [S(t)]=\frac{1}{\cosh [S(t)]} \frac{d}{d t} \cosh [S(t)]=\frac{d}{d t} \ln \{\cosh [S(t)]\}
$$

Hence, up to some unessential additive constant, we get the cost function

$$
G\left(\varepsilon_{1}, \varepsilon_{2}, \ldots, \varepsilon_{n}\right)=\frac{1}{2} \sum_{k} \varepsilon_{k}^{2}(t)-\frac{a}{n} \ln \left\{\cosh \left[\sum_{k} \varepsilon_{k}(t)\right]\right\} .
$$

\subsection{The guru function and the identity function}

As mentioned in Remark 1(ii), the guru function with the guru $\widetilde{k}$ can be represented by

$$
\forall j \neq \widetilde{k}, p_{i j}=0, p_{i \widetilde{k}}=1 .
$$

The system of differential equations given in (15) that we need to solve becomes

$$
\frac{d e_{i}}{d t}+e_{i}=\tanh \left(a e_{\widetilde{k}}\right), \quad i=1, \ldots, n .
$$

One can see that, on the one hand, the guru evolves only according to its own inclinations, and on the other hand, for all the remaining agents the guru evolution acts as a forcing term. The respective evolution equations of the agents differ only in their respective initial values. Hence, the following Proposition 2 is coherent with the intuition:

Proposition 2 If agents make their decisions according to the guru function, then the decision of each agent converges to the inclination of the guru.

Proof: Let $g_{0} \neq 0$ be a stable fixed point of the equation $\frac{d y}{d t}+y=a \tanh (y)$ (it exists by virtue of Lemma 1). This means that asymptotically (in long term) we can write, letting $g=e_{\widetilde{k}}$ for simplicity:

$$
g(t)=g_{0}+\gamma(t)
$$

where $p g_{0}=\tanh \left(g_{0}\right), p=\frac{1}{a}$, and $\gamma(t)$ converges to zero with $t$ tending to infinity. There exists $t_{0}$ such that for $t>t_{0}, \gamma(t)<\frac{\epsilon}{p g_{0}}$. For each agent $i$, we have

$$
\begin{aligned}
\frac{d e_{i}}{d t}+e_{i} & =\frac{1}{p} \tanh [g(t)]=\frac{1}{p} \tanh \left[g_{0}+\gamma(t)\right] \\
& =\frac{1}{p} \frac{\tanh \left(g_{0}\right)+\tanh [\gamma(t)]}{1+\tanh \left(g_{0}\right) \tanh [\gamma(t)]} \approx \frac{1}{p} \frac{p g_{0}+\gamma(t)}{1+p g_{0} \gamma(t)} \\
& \approx \frac{1}{p}\left[p g_{0}+\gamma(t)\right]\left[1-p g_{0} \gamma(t)\right] \\
& \approx \frac{1}{p}\left(p g_{0}-\left(p g_{0}\right)^{2} \gamma(t)+\gamma(t)\right) \\
& =g_{0}+B \gamma(t)
\end{aligned}
$$

where we used that $\frac{1}{1+\epsilon}=1-\epsilon(|\epsilon| \ll 1)$. 
The value $B$ is not important. The change of the function $e_{i}(t)=g_{0}+z_{i}(t)$ (the aim is to show that $z_{i}(t)$ converges to 0$)$ gives

$$
\frac{d z_{i}}{d t}+z_{i}=B \gamma(t)
$$

We will show that $\lim _{t \rightarrow \infty} z_{i}(t)=0$. Let us consider the equation

$$
\frac{d z}{d t}+z(t)=B \gamma(t)
$$

The homogeneous solution is

$$
z(t)=z_{0} \exp (-t)
$$

For $t>t_{0}$ the particular solution is of the form

$$
z(t)=u(t) \exp (-t)
$$

and it must satisfy

$$
\begin{gathered}
\frac{d z}{d t}+z(t)=\frac{d u}{d t} \exp (-t) \\
\frac{d u}{d t}=B \gamma(t) \exp (t)
\end{gathered}
$$

We have

$$
u(t)=B \int_{t_{0}}^{t} \gamma(u) \exp (u) d u
$$

The general solution is therefore of the form

$$
z(t)=z_{0} \exp (-t)+u(t) \exp (-t)=z_{0} \exp (-t)+B \exp (-t) \int_{t_{0}}^{t} \gamma(u) \exp (u) d u
$$

Asymptotically, the exponential disappears and

$$
z(t)=B \exp (-t) \int_{t_{0}}^{t} \gamma(u) \exp (u) d u
$$

Hence,

$$
\begin{gathered}
\left|\int_{t_{0}}^{t} \gamma(u) \exp (u) d u\right| \leq\left|\frac{\epsilon}{p g_{0}} \int_{t_{0}}^{t} \exp (u) d u\right| \\
\left|\int_{t_{0}}^{t} \gamma(u) \exp (u) d u\right| \leq \frac{\epsilon}{p g_{0}}\left[\exp (t)-\exp \left(t_{0}\right)\right]
\end{gathered}
$$

and therefore

$$
|z(t)| \leq \frac{B \epsilon}{p g_{0}} \exp (-t)\left[\exp (t)-\exp \left(t_{0}\right)\right]=\frac{B \epsilon}{p g_{0}}\left[1-\exp \left(t_{0}-t\right)\right]
$$

and

$$
|z(t)| \leq \frac{B \epsilon}{p g_{0}} .
$$


Since this converges to zero, we have for each $i$,

$$
e_{i}(t)=g_{0}+z_{i}(t) \rightarrow g_{0}
$$

As mentioned in Remark 1(iii), the identity function can be represented by $p_{i j}=\delta_{i j}$. The system of differential equations that we need to solve for each $i$ is

$$
\frac{d e_{i}}{d t}+e_{i}=\tanh \left(a e_{i}\right)
$$

Proposition 3 If agents make their decisions according to the identity function, then the decision of each agent converges to his own inclination.

Proof: Evident from Lemma 1.

\section{Followers in the dynamic model}

As recapitulated in Section 2.1, followers of a coalition $S$ are the agents who always follow the inclination of $S$, assuming that $S$ is unanimously inclined. The set of followers of coalition $S$ under the influence function $B$ is therefore defined as

$$
F_{B}(S):=\left\{i \in N: \forall e \in E_{S}\left[B_{i}(e)=e_{S}\right]\right\}
$$

where in our model, $B_{i}(e)=\operatorname{sgn}\left(\sum_{j} p_{i j} e_{j}\right)$.

In order to determine the followers for every influence function $B$, first we consider a particular case with $S=\left\{e_{1}\right\}$. Agent $i$ is the follower of $S$ if and only if

$$
\begin{gathered}
\forall e \in E, \operatorname{sgn}\left(\sum_{j} p_{i j} e_{j}\right)=\operatorname{sgn}\left(e_{1}\right) \\
\Leftrightarrow \forall e \in E, e_{1} \sum_{j} p_{i j} e_{j}>0 \\
\Leftrightarrow \forall e \in E, p_{i 1}+e_{1} \sum_{j \geq 2} p_{i j} e_{j}>0 \\
\Leftrightarrow p_{i 1}>\sum_{j \geq 2}\left|p_{i j}\right|
\end{gathered}
$$

Proposition 4 Agent $i$ is the follower of coalition $S$ if and only if

$$
\sum_{j \in S} p_{i j}>\sum_{j \notin S}\left|p_{i j}\right|
$$


Proof: In the general case, agent $i$ is the follower of $S$ if and only if

$$
\begin{gathered}
\forall e \in E_{S}, \operatorname{sgn}\left(\sum_{j} p_{i j} e_{j}\right)=\operatorname{sgn}\left(e_{k}\right)=\operatorname{sgn}\left(e_{l}\right)=\ldots \text { for each } e_{k}, e_{l} \in S \\
\Leftrightarrow \forall e \in E_{S}, e_{k} \sum_{j} p_{i j} e_{j}>0 \\
\Leftrightarrow \forall e \in E_{S}, \sum_{j \in S} p_{i j}+e_{k} \sum_{j \notin S} p_{i j} e_{j}>0 \\
\Leftrightarrow \sum_{j \in S} p_{i j}>\sum_{j \notin S}\left|p_{i j}\right|
\end{gathered}
$$

Note that for $|S|=1$, if $p_{i k}<0$ for each $i$, then $F_{B}\left(e_{k}\right)=\emptyset$.

Proposition 4 gives a very simple condition to test whether an agent is a follower of a coalition $S$ : the algebraic sum of the weights (that is, negative influence acts as a discounting factor) for the agents in $S$ should be greater than the sum of the absolute values of weights (that is, positive and negative influence are not distinguished) for the other agents.

Let us apply Proposition 4 to the majority influence function with $t=\left\lfloor\frac{n}{2}\right\rfloor+1$. We have $p_{i j}=\frac{1}{n}$ for each $i, j$. For each $S$, agent $i$ is the follower of coalition $S$ if and only if

$$
\sum_{j \in S} p_{i j}>\sum_{j \notin S}\left|p_{i j}\right| \Leftrightarrow \frac{|S|}{n}>\frac{n-|S|}{n} \Leftrightarrow|S|>\frac{n}{2} .
$$

This shows that in case of the majority influence function, the followers of $S$ depend only on the cardinality of $S$ and

$$
F_{\mathrm{Maj}^{[t]}}(S)= \begin{cases}N, & \text { if }|S|>\frac{n}{2} \\ \emptyset, & \text { if }|S| \leq \frac{n}{2}\end{cases}
$$

which is coherent with (1).

Let player $\widetilde{k}$ be the guru. We have then for each $i$

$$
p_{i j}= \begin{cases}0 & \text { if } j \neq \widetilde{k} \\ 1 & \text { if } j=\widetilde{k}\end{cases}
$$

Hence,

$$
\sum_{j \in S} p_{i j}=\left\{\begin{array}{lll}
1 & \text { if } & \widetilde{k} \in S \\
0 & \text { if } & \widetilde{k} \notin S
\end{array}, \quad \sum_{j \notin S}\left|p_{i j}\right|=\left\{\begin{array}{lll}
0 & \text { if } & \widetilde{k} \in S \\
1 & \text { if } & \widetilde{k} \notin S
\end{array}\right.\right.
$$

and therefore

$$
\sum_{j \in S} p_{i j}>\sum_{j \notin S}\left|p_{i j}\right| \Leftrightarrow \widetilde{k} \in S
$$


This result shows that in case of the guru function, the followers of $S$ depend only on the presence of the guru in coalition $S$. We get then

$$
F_{\text {Gur }[\widetilde{k}]}(S)= \begin{cases}N, & \text { if } \widetilde{k} \in S \\ \emptyset, & \text { if } \widetilde{k} \notin S\end{cases}
$$

which is equal to (2).

For the identity function $p_{i j}=\delta_{i j}$ for each $i, j$. We have

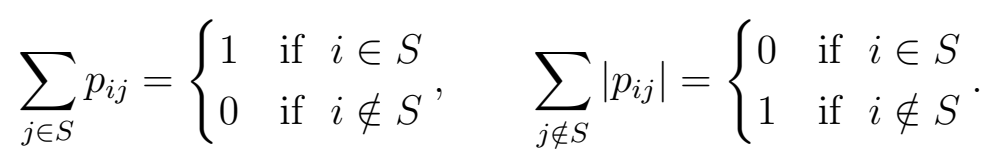

Hence,

$$
\sum_{j \in S} p_{i j}>\sum_{j \notin S}\left|p_{i j}\right| \Leftrightarrow i \in S .
$$

This means that the followers of $S$ under the identity function are the players of $S$ and only them, i.e.,

$$
F_{\text {Id }}(S)=S
$$

which confirms (3).

\section{Boss and approval sets in the dynamic model}

Using our dynamic model, we can also determine the boss and approval sets of command games. We have the following:

Proposition 5 (i) $S \subseteq N \backslash i$ is the boss set for agent $i$ if and only if

$$
\forall e \in E, \sum_{j \in S} p_{i j}>\sum_{j \notin S}\left|p_{i j}\right| .
$$

(ii) $S \subseteq N \backslash i$ is the approval set for agent $i$ if and only if

$$
\forall e \in E,\left\{\begin{array}{l}
\sum_{j \in S} p_{i j}+p_{i i}>\sum_{i \neq j \notin S}\left|p_{i j}\right| \\
\sum_{j \in S} p_{i j} \leq \sum_{j \notin S}\left|p_{i j}\right|
\end{array}\right.
$$

Proof: It is clear from the proof of Proposition 4.

Proposition 5 provides simple conditions for determining boss sets and approval sets. Boss sets for $i$ are those sets $S$ such that the algebraic sum of weights for agents in $S$ is greater than the sum of absolute values for the other agents. From Proposition 4, we see that an agent follows his boss sets. The condition for approval sets shows that roughly speaking, an approval set of $i$ together with $i$ has "more weight" than the remaining agents, but it is not the case anymore if $i$ 's weight is counted for the weights of agents outside the approval set. 
Let us apply Proposition 5 to the majority influence function with $t=\left\lfloor\frac{n}{2}\right\rfloor+1$. We have $p_{i j}=\frac{1}{n}$ for each $i, j . S$ is the boss set for agent $i$ if and only if

$$
\sum_{j \in S} p_{i j}>\sum_{j \notin S}\left|p_{i j}\right| \Leftrightarrow \frac{|S|}{n}>\frac{n-|S|}{n} \Leftrightarrow|S|>\frac{n}{2}
$$

We have therefore

$$
\operatorname{Boss}_{i}^{\mathrm{Maj}^{[t]}}=\left\{S \subseteq N \backslash i:|S|>\frac{n}{2}\right\}
$$

which is coherent with (4).

$S$ is the approval set for agent $i$ if and only if

$$
\forall e \in E,\left\{\begin{array} { l } 
{ \sum _ { j \in S } p _ { i j } + p _ { i i } > \sum _ { i \neq j \notin S } | p _ { i j } | } \\
{ \sum _ { j \in S } p _ { i j } \leq \sum _ { j \notin S } | p _ { i j } | }
\end{array} \Leftrightarrow \left\{\begin{array}{l}
\frac{|S|}{n}+\frac{1}{n}>\frac{n-|S|-1}{n} \\
\frac{|S|}{n} \leq \frac{n-|S|}{n}
\end{array}\right.\right.
$$

We have therefore

$$
\operatorname{App}_{i}^{\mathrm{Maj}^{[t]}}=\left\{S \subseteq N \backslash i:|S|=\left\lfloor\frac{n}{2}\right\rfloor\right\}
$$

which is coherent with (5).

Let $\widetilde{k}$ be the guru. By virtue of Proposition 5(i) and (19), we have

$$
\operatorname{Boss}_{\widetilde{k}}^{\text {Gur }}{ }^{[\widetilde{k}]}=\emptyset \text { and } \operatorname{Boss}_{k}^{\text {Gur }}{ }^{[\widetilde{k}]}=\{S \subseteq N \backslash k: \widetilde{k} \in S\} \text { for } k \neq \widetilde{k}
$$

Using Proposition 5(ii), we conclude that $S$ is the approval set for agent $i$ if and only if $p_{i i}=p_{\widetilde{k} \widetilde{k}}=1$. Hence, every set not containing $\widetilde{k}$ is the approval set for $\widetilde{k}$, and every agent different from $\widetilde{k}$ has the empty approval set:

$$
A p p_{\widetilde{k}}^{\mathrm{Gur}^{[\widetilde{k}]}}=2^{N \backslash \widetilde{k}} \text { and } A p p_{k}^{\text {Gur }}{ }^{[\widetilde{k}]}=\emptyset \text { for } k \neq \widetilde{k}
$$

We get therefore the results given in (6) and (7).

For the identity function, we have $p_{i j}=\delta_{i j}$ for each $i, j$. Using Proposition 5(i) and (20), we get $\operatorname{Boss}_{i}^{\text {ld }}=\emptyset$. By virtue of Proposition 5(ii), we conclude that $S$ is the approval set for agent $i$ if and only if $p_{i i}=1$, that is, each set not containing $i$ is the approval set for $i$. Hence, $A p p_{i}^{\text {ld }}=2^{N \backslash i}$, which gives exactly (8).

\section{Conclusions}

The paper concerns the influence model originally introduced in Hoede and Bakker (1982) and later studied and generalized in several works. We have proposed a new approach to analyze the dynamic of the model. This approach is based on the use of differential equations.

To be more precise, we have introduced a dynamic model of influence in which an agent may give a certain importance to other agents in making his final decision. This importance is reflected by a weight which, depending of the sign (positive, negative or zero), corresponds to the stimulation, the inhibition or the absence of relation, respectively. We have defined the exhortation obtained by an agent as the weighted sum of the 
opinions that the agent receives from the others. The updating rule is based on the sign of the exhortation: its positive (negative) value means going to the positive (negative) state, and the exhortation equal to zero corresponds to staying in the present state.

The main ideas of the application of this approach to the model of influence is to switch to the continuum case and to apply some approximations allowing the use of differential equations systems. The solutions of these systems give the same results obtained earlier for the classical influence functions. It also leads to the results on followers obtained for the discrete case, and to the results on the boss and approval sets of command games. In the paper we have shown that if the majority function is used, then the decision of each agent converges to the sign of the sum of the agents' inclinations, and in case of the guru function, the decision of each agent converges to the inclination of the guru. Under the identity function, the agents' decisions converge obviously to their own inclinations. Furthermore, we have described the necessary and sufficient condition that an agent is the follower of a coalition in the dynamic framework. We have used that result to determine the sets of followers for the majority function, the guru function, and the identity function. We have also determined the necessary and sufficient conditions that a coalition is the boss set or the approval set for an agent in the dynamic model. We have applied these results to determine the boss and approval sets of command games equivalent to the three influence functions in question: the majority influence function, the guru function, and the identity function. Both in the case of followers and boss and approval sets, the conditions depend on the relation(s) between the weights that reflect the importance given by the agents to the inclinations of the others in making their own decisions.

To the best of our knowledge, the differential equations approach has never been applied before to the influence model in question and is also innovative with respect to analysis of the influence framework in general. We believe that this approach can bring new insights in the analysis of the dynamic aspects. Moreover, modeling positive and negative influence in the same time by incorporating the importance weights that can be of any sign increases significantly the applicability of the model.

In the paper, we aimed at introducing this new and useful framework of influence and at studying the dynamic of the standard influence functions defined in the one-step yesno model of influence. Although we have focused on these classical influence functions, we would like to stress that the approach allows the study of new concepts, like e.g. the weighted majority function. In our future research on this model, we would like to deliver a detailed analysis of the concepts that were not defined in the original yes-no model of influence, but could be defined in the present stimulation-inhibition framework. There are several possible research issues related to this model that could be raised. In the future research on this framework, we would like to analyze, in particular, non-linear influence functions and to consider temporary effects in the behavior of a group of agents. 


\section{Bibliography}

C. Asavathiratham. Influence model: a tractable representation of networked Markov chains. PhD thesis, Massachusetts Institute of Technology, Cambridge, MA, 2000.

C. Asavathiratham, S. Roy, B. Lesieutre, and G. Verghese. The influence model. IEEE Control Systems Magazine, 21:52-64, 2001.

R. L. Berger. A necessary and sufficient condition for reaching a consensus using DeGroot's method. Journal of the American Statistical Association, 76:415-419, 1981.

M. H. DeGroot. Reaching a consensus. Journal of the American Statistical Association, 69:118-121, 1974.

P. DeMarzo, D. Vayanos, and J. Zwiebel. Persuasion bias, social influence, and unidimensional opinions. Quarterly Journal of Economics, 118:909-968, 2003.

N. E. Friedkin and E. C. Johnsen. Social influence and opinions. Journal of Mathematical Sociology, 15:193-206, 1990.

N. E. Friedkin and E. C. Johnsen. Social positions in influence networks. Social Networks, 19:209-222, 1997.

B. Golub and M. O. Jackson. Naïve learning in social networks and the wisdom of crowds. American Economic Journal: Microeconomics, 2(1):112-149, 2010.

M. Grabisch and A. Rusinowska. Measuring influence in command games. Social Choice and Welfare, 33:177-209, 2009.

M. Grabisch and A. Rusinowska. A model of influence in a social network. Theory and Decision, 69(1):69-96, 2010a.

M. Grabisch and A. Rusinowska. A model of influence with an ordered set of possible actions. Theory and Decision, 69(4):635-656, $2010 \mathrm{~b}$.

M. Grabisch and A. Rusinowska. Different approaches to influence based on social networks and simple games. In A. van Deemen and A. Rusinowska, editors, Collective Decision Making: Views from Social Choice and Game Theory, Series Theory and Decision Library C, Volume 43, pages 185-209. Springer-Verlag Berlin Heidelberg, 2010c.

M. Grabisch and A. Rusinowska. Influence functions, followers and command games. Games and Economic Behavior, 72(1):123-138, 2011a.

M. Grabisch and A. Rusinowska. A model of influence with a continuum of actions. Journal of Mathematical Economics, 47:576-587, 2011b.

M. Grabisch and A. Rusinowska. A model of influence based on aggregation functions. CES Working Paper, 2011.58, ftp://mse.univ-paris1.fr/pub/mse/CES2011/11058.pdf, 2011c.

C. Hoede and R. Bakker. A theory of decisional power. Journal of Mathematical Sociology, 8:309-322, 1982.

X. Hu and L. S. Shapley. On authority distributions in organizations: equilibrium. Games and Economic Behavior, 45:132-152, 2003a.

X. Hu and L. S. Shapley. On authority distributions in organizations: controls. Games and Economic Behavior, 45:153-170, 2003b.

M. O. Jackson. Social and Economic Networks. Princeton University Press, 2008.

M. Koster, I. Lindner, and S. Napel. Voting power and social interaction. SING7 Conference, Palermo, July 7-9, 2010. 
U. Krause. A discrete nonlinear and nonautonomous model of consensus formation. In S. Elaydi, G. Ladas, J. Popenda, and J. Rakowski, editors, Communications in Difference Equations. Amsterdam: Gordon and Breach, 2000.

J. Lorenz. A stabilization theorem for dynamics of continuous opinions. Physica A, 355: 217-223, 2005.

E. Maruani. Jeux d'influence dans un réseau social. Mémoire de recherche, Centre d'Economie de la Sorbonne, Université Paris 1, 2010.

A. Rusinowska. Different approaches to influence in social networks. Invited tutorial for the Third International Workshop on Computational Social Choice (COMSOC 2010), Düsseldorf, available at http://ccc.cs.uni-duesseldorf.de/COMSOC2010/slides/invited-rusinowska.pdf, 2010. 